PROCEEDINGS OF THE

AMERICAN MATHEMATICAL SOCIETY

Volume 129, Number 6, Pages 1833-1842

S 0002-9939(00)05739-7

Article electronically published on October 31, 2000

\title{
ESTIMATING THE RATIONAL LS-CATEGORY OF ELLIPTIC SPACES
}

\author{
SONIA GHORBAL AND BARRY JESSUP \\ (Communicated by Ralph Cohen)
}

\begin{abstract}
An elliptic space is one whose rational homotopy and rational cohomology are both finite dimensional. We prove, for Toomer's invariant, two improvements of the estimate of the Mapping theorem relying on data from the homotopy Lie algebra of the space. In particular, we show that if $S$ is elliptic,
\end{abstract}

$$
\operatorname{cat}_{0} S \geq \operatorname{dim} L_{S}^{\mathrm{ev}}+\operatorname{dim} Z L_{S}^{\text {odd }},
$$

where $L_{S}$ is the rational homotopy Lie algebra of $S$ and $Z L_{S}$ its centre. Several interesting examples are presented to illustrate our results.

\section{INTRODUCTION}

The Lusternik-Schnirelmann category of a space $S$ is the least number of contractible (in $S$ ) open sets needed to cover $S$, minus one. It is a rather subtle homotopy invariant which is difficult to compute, except in special cases where it agrees with other simpler invariants, such as the dimension or the cup length of the cohomology ring. The difficulties are attenuated somewhat for rational spaces, where Felix and Halperin [FH] used Sullivan models to provide a tractable algebraic characterization of the L-S category of the rationalization $S_{\mathrm{Q}}$ of a simply connected CW complex.

However, even if one is given an elliptic minimal model, where the model data and the cohomology are both finite, and for which recent results [FHL] reduce the calculation of $\operatorname{cat}_{0} S:=\operatorname{cat}_{\mathbf{Q}_{\mathbf{Q}}}$ considerably, one must still find a "longest" representative of the top cohomology class, something which is non-trivial in examples with more than a few generators. Some formulae are available in very special cases J1] but useful estimates are still important, and we will provide some new lower bounds which depend on simple invariants of the rational homotopy Lie algebra of $S$.

Received by the editors September 15, 1999.

2000 Mathematics Subject Classification. Primary 55P62.

Key words and phrases. Elliptic spaces, minimal models, rational category.

This research was partially supported by a UCL postdoctoral grant, the University of Ottawa and the National Science and Engineering Research Council of Canada.

The second author also acknowledges the support of the National Science and Engineering Research Council of Canada. 
Our method is to use the Postnikov tower of a space $S$, building up $S$ by fibrations over one (rational) homotopy class at a time. At each step, we will have a fibration $F \rightarrow E \stackrel{p}{\rightarrow} K(\mathbf{Q}, n)$ with zero connecting homomorphsim, where $E$ is some highly connected cover of $S$. We then estimate cat ${ }_{0} E$ in terms of cat ${ }_{0} F$ by imposing some restrictions on the homotopy Lie algebra of $E$ involving the pre-image under $p$ of the fundamental class $\iota_{n}$ of $K(\mathbf{Q}, n)$. Since the Postnikov fibre $F$ is the total space of the next step, the method will terminate if $S$ has finite total rational homotopy. Since $\operatorname{cat}_{0} S<\infty$ and $\operatorname{dim} \pi_{*} S \otimes \mathbf{Q}<\infty$ characterize $S$ as elliptic [FHT], this partially explains our interest in elliptic spaces.

The first result relating cat $_{0} E$ and $\operatorname{cat}_{0} F$ in this situation was the Mapping theorem [FH], which implies in particular that if $F \rightarrow E \stackrel{p}{\rightarrow} K(Q, n)$ is a rational fibration with zero connecting homomorphism in rational homotopy, then cat $_{0} E \geq$ $\operatorname{cat}_{0} F$. Then, in [J], this was generalized to show that if $n$ is odd and the holonomy action of the base on the homology of the fibre is locally nilpotent, one can improve the estimate to cat $_{0} E \geq \operatorname{cat}_{0} F+1$. Indeed, in this result there is already a clue that the homotopy Lie algebra may be implicated in these matters.

Recall that for a space $S$, the rational homotopy Lie algebra of $S$ is the graded Lie algebra whose underlying graded vector space is

$$
L_{S}^{*}:=\pi_{*} \Omega S \otimes \mathbf{Q} \stackrel{\Omega}{\cong} \pi_{*+1} S \otimes \mathbf{Q},
$$

where $\Omega S$ denotes the space of based loops on $S$, and we have also denoted the isomorphism $\pi_{*+1} S \cong \pi_{*} \Omega S$ by $\Omega$. The Lie bracket is the Samelson product [,] : $L_{S}^{p} \otimes L_{S}^{q} \rightarrow L_{S}^{p+q}$, which may be defined using either the commutator of loops W] p.467] or the Whitehead product [W, p.472].

It was noted in [J, §3] that if the homotopy class $\Omega\left(p_{*}\right)^{-1} \iota_{n}$ acts locally nilpotently in $L_{E}$ in the fibration $F \rightarrow E \stackrel{p}{\rightarrow} K(Q, n)$, and $n$ is odd, then cat $_{0} E \geq$ cat $_{0} F+1$. The first of our theorems for even $n$ is in this same spirit. To motivate the second, consider the fibrations

$$
\mathbf{S}^{3} \rightarrow \mathbf{S}^{2} \stackrel{q}{\rightarrow} K(\mathbf{Z}, 2)
$$

and

$$
\mathbf{S}^{5} \rightarrow \mathbf{C P}^{2} \stackrel{r}{\rightarrow} K(\mathbf{Z}, 2),
$$

where both $q$ and $r$ are chosen to represent the generators of the cohomology groups in dimension 2. In (1), $\operatorname{cat}_{0} \mathbf{S}^{2}=\operatorname{cat}_{0} \mathbf{S}^{3}=1$ while in (2), $\operatorname{cat}_{0} \mathbf{C P}^{2}=\operatorname{cat}_{0} \mathbf{S}^{5}+1$. One of the differences between these examples is that the fundamental homotopy class $\iota_{2}$ of the base satisfies $\left[\Omega q_{*}^{-1} \iota_{2}, \Omega q_{*}^{-1} \iota_{2}\right] \neq 0$ in $\pi_{2} \Omega \mathbf{S}^{2}$, while $\left[\Omega r_{*}^{-1} \iota_{2}, \Omega r_{*}^{-1} \iota_{2}\right]=0$ in $\pi_{2} \Omega \mathbf{C P}^{2}=0$. While we present an example later to show that $\left[\Omega p_{*}^{-1} \iota_{n}, \Omega p_{*}^{-1} \iota_{n}\right]=0$ is not sufficient to guarantee that cat $_{0} E \geq$ cat $_{0} F+1$, our second theorem gives some extra conditions under which this will suffice.

To avoid unhelpful notation, when we have a fibration $F \rightarrow E \stackrel{p}{\rightarrow} K(\mathbf{Q}, n)$ with zero connecting homomorphism, we will identify $\Omega p_{*}^{-1} \iota_{n}$ with $\iota_{n}$, and say that $\iota_{n}$ is in the centre of $L_{E}$ if $\Omega p_{*}^{-1} \iota_{n}$ is.

In fact, the results we obtain here are really about Toomer's invariant $\mathrm{e}_{0}[\mathrm{~T}]$, which we define in the next section, but the equality cat ${ }_{0}=e_{0}$ for Poincaré duality complexes established by Félix, Halperin and Lemaire FHL imply the same for cat $_{0}$ in the case of elliptic spaces. 
Theorem 1. Suppose $n$ is even, $F \rightarrow E \rightarrow K(\mathbf{Q}, n)$ is a rational fibration with zero connecting homomorphism in rational homotopy, and that $H^{*}(F ; \mathbf{Q})$ satisfies Poincaré duality. If the fundamental homotopy class of the base is in the centre of the rational homotopy Lie algebra of $E$, we have

$$
\mathrm{cat}_{0} E \geq \mathrm{cat}_{0} F+1 \text {. }
$$

We can relax the hypothesis on the fundamental homotopy class of the base somewhat and still obtain the same result if we further restrict the fibre.

Theorem 2. Suppose $n$ is even, $F \rightarrow E \rightarrow K(\mathbf{Q}, n)$ is a rational fibration with zero connecting homomorphism in rational homotopy, and $F$ is a coformal Poincaré duality complex. If the fundamental homotopy class $\iota_{n}$ of the base satisfies $\left[\iota_{n}, \iota_{n}\right]=$ 0 in $\pi_{*}(E)$, we have

$$
\operatorname{cat}_{0} E \geq \operatorname{cat}_{0} F+1 .
$$

We remark that these theorems improve the estimates of [FH] Theorem 10.4(iv)].

This paper is organized as follows. The next section summarizes just as much rational homotopy theory as we will need, along with the characterizations of cat ${ }_{0}$ and Toomer's invariant in terms of Sullivan minimal models. We then prove Theorems 1 and 2, and the last section gives some corollaries and examples to illustrate the utility of our results.

\section{RATIONAL HOMOTOPY AND cat $_{0}$}

All our spaces will be simply connected spaces with the homotopy type of CW complexes with rational cohomology of finite type. We will work with $\mathbf{Q}$ as ground field and our principal tools are Sullivan models. A detailed description of these and the standard tools of rational homotopy can be found in $[\mathrm{BG},[\mathrm{F}],[\mathrm{H}]$ and $[\mathrm{S}]$. For our purposes, the following will suffice.

A commutative graded differential algebra (hereafter cgda) $\left(A, d_{A}\right)$ is c-connected if $H^{0}\left(A, d_{A}\right) \cong \mathbf{Q}$. A morphism $\phi:\left(A, d_{A}\right) \rightarrow\left(B, d_{B}\right)$ is a quasi-isomorphism if $H^{*} \phi$ is an isomorphism. If $\phi:\left(A, d_{A}\right) \rightarrow\left(B, d_{B}\right)$ is a morphism of c-connected cgda's, a Sullivan model of $\phi$ is a factoring $\phi=\psi i$ in

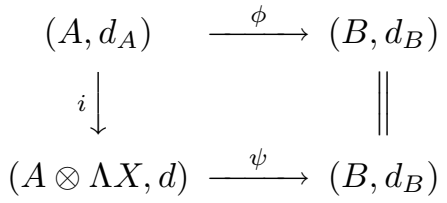

where $i(a)=a \otimes 1$ for $a \in A$ and $\psi$ is a quasi-isomorphism. Here, as elsewhere, $\Lambda X$ denotes the free commutative-graded algebra on the graded vector space $X=$ $\sum_{n \geq 0} X^{n}$ which has a well ordered, homogeneous basis $\left\{x_{\alpha}\right\}$ such that, if $X_{<\alpha}$ denotes $\operatorname{span}\left\{x_{\beta} \mid \beta<\alpha\right\}$, we have

(1) $d x_{\alpha} \in A \otimes \Lambda X_{<\alpha}$ and

(2) $\alpha<\beta \Longrightarrow \operatorname{deg} \alpha \leq \operatorname{deg} \beta$.

If $X=X^{\geq 2}$, (1) and (2) are equivalent to $d x_{\alpha} \in \Lambda^{\geq 2}\left(X_{<\alpha}\right)$. Such a basis is called a $K$-S basis. The factoring is determined up to isomorphism by $\phi$ and we say that $i$ represents $\phi$.

The projection $A \otimes \Lambda X \stackrel{\rho}{\rightarrow} A \otimes \Lambda X / I \cong \Lambda X$ (in which $I$ denotes the differential ideal generated by the augmentation ideal of $A$ ) induces a differential $\tilde{d}$ in $\Lambda X$ and the sequence $\left(A, d_{A}\right) \stackrel{i}{\rightarrow}(A \otimes \Lambda X, d) \stackrel{\rho}{\rightarrow}(\Lambda X, \tilde{d})$ is called a minimal $K-S$ extension. 
Sullivan defined a contravariant functor $\mathcal{A}$ which associates to each space $S$ a cgda $\mathcal{A}(S)$ over $\mathbf{Q}$ which computes the rational homotopy of $S$. A Sullivan minimal model of $(\mathbf{Q}, 0) \rightarrow \mathcal{A}(S)$ is of the form $(\mathbf{Q}, 0) \rightarrow(\Lambda X, d) \rightarrow \mathcal{A}(S)$ and $(\Lambda X, d)$ is called the (Sullivan) minimal model of $S$, and it is unique up to isomorphism. If $S \rightarrow T$ is a continuous map, a standard lifting lemma applied to $\mathcal{A}(f)$ gives a unique homotopy class of morphisms between their minimal models, any of which is called a Sullivan representative of $f$.

For every Serre fibration $\xi: F \stackrel{i}{\rightarrow} E \stackrel{p}{\rightarrow} B$, there is a commutative diagram of augmented cgda's

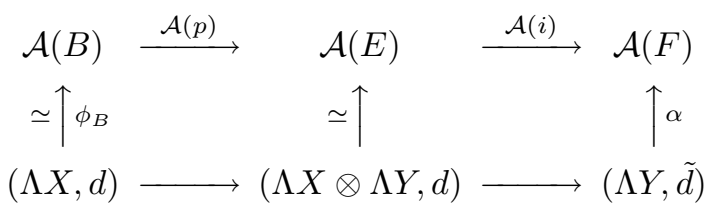

in which $(\Lambda X, d)$ is a Sullivan model for $B$, and the bottom row is the minimal model of $\mathcal{A}(p) \circ \phi_{B}$. If $\alpha:(\Lambda Y, \tilde{d}) \rightarrow \mathcal{A}(F)$ is a quasi-isomorphism, $\xi$ is called a rational fibration. In particular, if $B$ is simply connected, $\xi$ is a rational fibration G]. We call the bottom row of this diagram a minimal K-S extension associated to the fibration. Note that in general, the middle cgda need not be a minimal model of $E$. It will be, precisely when the image of the connecting homomorphism $\partial: \pi_{k}(B) \rightarrow \pi_{k-1}(F)$ is strictly torsion (see [1] $)$.

The minimal model of $S$ carries the rational homotopy type of $S$; that is, the homotopy type of its localization at the rationals $S_{\mathbf{Q}}$. In particular, as graded vector spaces, $\hat{X}:=\operatorname{Hom}(X, \mathbf{Q}) \cong \pi(S) \otimes \mathbf{Q}$. Moreover, if we define $(s \hat{X})^{n}:=\hat{X}^{n+1}$ for $n>0$, then $(s \hat{X})^{n}$ is isomorphic to $\pi_{n}(\Omega S) \otimes \mathbf{Q}$, and the homotopy Lie algebra of $S$ is encoded in the minimal model $(\Lambda X, d)$ as follows.

The differential $d$ can be written as a sum of derivations $d=d_{2}+d_{3}+\ldots$ where $d_{i}: X \rightarrow \Lambda^{i} X$. The fact that $d^{2}=0$ implies the same for $d_{2}$. The dual of $d_{2}: X \rightarrow \Lambda^{2} X$ induces a bilinear and antisymmetric map $L: \hat{X} \otimes \hat{X} \rightarrow \hat{X}$, which represents the Samelson product in $\pi(\Omega S) \otimes \mathbf{Q}$ via

$$
[s \hat{x}, s \hat{y}]=s L(\hat{x} \otimes \hat{y}),
$$

where we have denoted by $s$ the isomorphism $\hat{X} \cong s \hat{X}$, which is of degree -1 . The Jacobi identity for [,] is equivalent to $d_{2}^{2}=0$. Henceforth, we shall also call $s \hat{X}$ with this product the (rational) homotopy Lie algebra of $S$.

In particular, if $\left\{x_{\alpha}\right\}$ is a K-S basis for $X$, and $\left\{\hat{x}_{\alpha}\right\}$ its dual basis, then

1. $\left[s \hat{x}_{\alpha}, s \hat{x}_{\beta}\right]=0$ iff the coefficient of $x_{\alpha} x_{\beta}$ in $d_{2} x_{\gamma}$ is zero for all $\gamma$, so that

2. $s \hat{x}_{\alpha}$ is in the centre of the homotopy Lie algebra iff

$$
d_{2}: X \rightarrow \Lambda^{2}\left\langle x_{\beta} \mid \beta \neq \alpha\right\rangle .
$$

Where no confusion will arise, we shall say that $x_{\alpha}$ belongs to the centre of $L_{S}$ if $s \hat{x}_{\alpha}$ does.

A space $S$ is coformal if $d=d_{2}$ in a minimal model of $S$. In this case, the rational homotopy type of $S$ is completely determined by its rational homotopy Lie algebra. Moreover, a space is elliptic iff $\operatorname{dim} X$ and $\operatorname{dim} H(\Lambda X, d)$ are both finite in any minimal model.

We now briefly recall the description of rational L-S category and Toomer's invariant in terms of Sullivan models. Let $S$ be a space and $(\Lambda X, d)$ a minimal 
model of $S$. Consider the projection $\Lambda X \rightarrow \Lambda X / \Lambda^{>m} X$. This induces a differential $D$ in $\Lambda X / \Lambda^{>m} X$ which makes the projection a map of differential algebras. Let

$$
(\Lambda X, d) \rightarrow(\Lambda X \otimes \Lambda V, d) \stackrel{\simeq}{\rightarrow}\left(\Lambda X / \Lambda^{>m} X, D\right)
$$

be a minimal model for the projection.

The rational category of $(\Lambda X, d)$, denoted cat ${ }_{0} S$, is the least $m$ such that there is a map $r:(\Lambda X \otimes \Lambda V, d) \rightarrow(\Lambda X, d)$ of cgdas which satisfies $r(x)=x$ for all $x \in X$. Félix and Halperin $[\mathrm{FH}]$ proved that $\operatorname{cat}_{0} S=\operatorname{cat} S_{\mathbf{Q}}$.

Toomer's invariant, denoted $\mathrm{e}_{0} S$, may be defined as the least $m$ such that there is a map $r:(\Lambda X \otimes \Lambda V, d) \rightarrow(\Lambda X, d)$ of graded differential vector spaces which satisfies $r(x)=x$ for all $x \in X$. It is straightforward that

$$
e_{0} S=\sup \left\{k \mid \exists \alpha \in \Lambda^{\geq k} X \text { with } 0 \neq[\alpha] \in H^{*} S\right\} .
$$

Clearly, $e_{0} S \leq \operatorname{cat}_{0} S$ and it is known that coformal spaces [FH] p.30] and Poincaré duality spaces [FHL] satisfy cat $_{0}=\mathrm{e}_{0}$. In particular, since elliptic spaces are Poincaré duality spaces [H1, p.188], they also satisfy cat $_{0}=\mathrm{e}_{0}$.

\section{Proofs OF THE THEOREMS}

With the notions and notation of the previous section, Theorems 1 and 2 now follow directly from the following:

Proposition 1. Suppose the degree of a is even, $\Lambda(a ; 0) \rightarrow \Lambda(a, X ; d) \rightarrow \Lambda(X ; \bar{d})$ is a minimal $K-S$ extension with zero connecting homomorphism, and that $H^{*}(\Lambda X ; \bar{d})$ satisfies Poincare duality. Then, if a is in the centre of the homotopy Lie algebra of $\Lambda(a, X ; d)$, we have

$$
\operatorname{cat}_{0} \Lambda(a, X ; d) \geq \mathrm{e}_{0} \Lambda(X ; \bar{d})+1 .
$$

Proposition 2. Suppose the degree of a is even, $\Lambda(a ; 0) \rightarrow \Lambda(a, X ; d) \rightarrow \Lambda(X ; \bar{d})$ is a minimal K-S extension with zero connecting homomorphism. Suppose further that $\bar{d}=\bar{d}_{2}$, and that $H^{*} \Lambda(X ; \bar{d})$ is a Poincaré duality algebra. Then, if $[a, a]=0$, we have

$$
\operatorname{cat}_{0} \Lambda(a, X ; d) \geq \mathrm{e}_{0} \Lambda(X ; \bar{d})+1 .
$$

Proof of Proposition 1. First note that we can write $d=\bar{d}+\sum_{i} a^{i} \eta_{i}$, where each $\eta_{i}$ is a derivation of $\Lambda X$ of odd degree. The fact that $a$ is in the centre of the homotopy Lie algebra of $\Lambda(a, X ; d)$ implies that $\eta_{1}: \Lambda^{k} X \rightarrow \Lambda^{\geq k+1} X, \eta_{2}: \Lambda^{k} X \rightarrow \Lambda^{\geq k} X$ and $\eta_{j}: \Lambda^{k} X \rightarrow \Lambda^{\geq k-1} X$ for $j>2$. In particular, if $\beta \in \Lambda^{\geq e} X$ and we write $d \beta=\bar{d} \beta+a \beta_{1}$, then $\beta_{1} \in \Lambda^{\geq e+1}(a, X)$.

Since the fibre is a Poincaré duality space and the base is Gorenstein, by [FHT1. Thm. 4.3], the total space is also Gorenstein. Moreover, since we may assume that $\operatorname{cat}_{0} \Lambda(a, X ; d)<\infty$, by Theorem 3.1 of the same article, we conclude that $H^{*} \Lambda(a, X ; d)$ is a Poincaré duality algebra. Then, by Propositions 5.1 and 5.3, again of [FHT1], the formal dimension of the fibre is strictly greater than that of the total space.

Now suppose $e_{0} \Lambda(X ; \bar{d})=e$, and let $\beta \in \Lambda^{\geq e} X$ be a cycle representing a top class. Then, $d \beta=a \alpha$, where $d \alpha=0$ and $\alpha \in \Lambda^{\geq e+1}(a, X)$, by previous remarks. Suppose that $\alpha$ is exact in $\Lambda(a, X ; d)$, say $\alpha=d \gamma$. Then, $\beta-a \gamma$ is a $d$-cycle of degree greater than the formal dimension of $\Lambda(a, X ; d)$. Hence, $\beta-a \gamma=d z$ for some $z=z_{0}+a z_{1}$, where $z_{0} \in \Lambda X$. Comparison of coefficients of powers of $a$ on 
both sides of $\beta-a \gamma=d z$ yields $\bar{d} z_{0}=\beta$, a contradiction. Thus, the class of $\alpha$ is nonzero in $H^{*} \Lambda(a, X ; d)$, and so $e_{0} \Lambda(a, X ; d) \geq e+1$.

Proof of Proposition 2. This is much like the previous proof, but here the hypotheses on the homotopy Lie algebra imply only that $\eta_{1}: \Lambda^{k} X \rightarrow \Lambda^{\geq k} X, \eta_{2}: \Lambda^{k} X \rightarrow$ $\Lambda^{\geq k} X$ and $\eta_{j}: \Lambda^{k} X \rightarrow \Lambda^{\geq k-1} X$ for $j>2$.

Hence, if $e_{0} \Lambda(X ; \bar{d})=e$ and $\beta \in \Lambda^{\geq e} X$ is a cycle representing the top class, then we can write $d \beta=a \alpha_{0}+a^{2} \alpha_{1}$, where $d\left(\alpha_{0}+a \alpha_{1}\right)=0$ as before, and $\alpha_{0} \in \Lambda \geq e X$ and $a \alpha_{1} \in \Lambda^{\geq e+1}(a, X)$.

Now $d^{2}=0$ implies that $\bar{d} \alpha_{0}=0$, and $\left|\alpha_{0}\right|<|\beta|$, so if $\alpha_{0}$ were not a $\bar{d}$-boundary, using the Poincaré duality, we could multiply it up to the top class, and this would give a nonzero cohomology class in the fibre of length at least $e+1$, contrary to assumption. Since $\bar{d}$ increases the length of monomials by exactly one, we can choose $\epsilon \in \Lambda^{e-1} X$ with $\bar{d} \epsilon=\alpha_{0}$. Then, $d(\beta-a \epsilon)=a^{2} \alpha^{\prime}$ with $a \alpha^{\prime} \in \Lambda^{\geq e+1}(a, X)$. The proof that $a \alpha^{\prime}$ is a nonzero class in $H^{*} \Lambda(a, X ; d)$ now follows as before.

Remarks. The association $\beta \mapsto \alpha$ in both proofs above is just the connecting homomorphism of the long exact sequence obtained from $0 \rightarrow \Lambda(a, X ; d) \stackrel{\cdot a}{\rightarrow} \Lambda(a, X ; d) \stackrel{p}{\rightarrow}$ $\Lambda(X ; \bar{d}) \rightarrow 0$, where $p$ is the projection onto $\Lambda(X ; \bar{d})$. Note that the argument above (without any assumptions on $a$ ) also gives a simple Mapping theorem for $\mathrm{e}_{0}$ in this particular case.

The argument of Proposition 2 also yields the same conclusion if we replace the assumption of coformality by $l_{0}(F)=1$, where $l_{0}(F)$ is the rational Ginsburg invariant, namely the least $m$ for which the rational Milnor-Moore spectral sequence for $F$ collapses at the $(m+1)$ st stage. This implies that if $d \epsilon \in \Lambda^{\geq k+1} X$, we may assume that $\epsilon \in \Lambda^{\geq k} X$, so $\epsilon$ still has the desired length.

\section{Applications to ELLiptic SPACES AND EXAMPLES}

If $S$ is elliptic, and $F \rightarrow S \rightarrow B$ is a rational fibration with zero connecting homomorphism, then the Mapping theorem implies that $F$ will also be elliptic. Now let $L_{S}$ denote the rational homotopy Lie algebra of an elliptic space $S$, and $Z L_{S}$ its centre. An obvious induction using Theorem 1 and [J] yields

Theorem 3. If $E$ is elliptic and $F \rightarrow E \stackrel{p}{\rightarrow} B$ is a fibration with coker $\pi_{*}(p)$ strictly torsion, then

$$
\operatorname{cat}_{0} E \geq \operatorname{dim} L_{B}^{\mathrm{ev}}+\operatorname{dim} \pi_{*}(\Omega p) Z L_{E}^{\mathrm{odd}}+\operatorname{cat}_{0} F .
$$

In particular,

Corollary 1. If $S$ is elliptic, $\operatorname{cat}_{0} S \geq \operatorname{dim} L_{S}^{\mathrm{ev}}+\operatorname{dim} Z L_{S}^{\text {odd }}$.

Note that $\operatorname{cat}_{0} S \geq \operatorname{dim} L_{S}^{\mathrm{ev}}$ was proven in $[\mathrm{FH}]$.

Corollary 2. If $L_{S}^{\text {odd }} \subset Z L_{S}$ (e.g. if $L_{S}$ is abelian), then $\operatorname{cat}_{0} S \geq \operatorname{dim} L_{S}$.

It doesn't seem fruitful to inductively apply Theorem 2 to an elliptic space $E$, since if $F$ is coformal in $F \rightarrow E \rightarrow K(\mathbf{Q}, 2 n)$, no element of $L_{F}^{\text {odd }}$ can satisfy $[a, a]=0$ (because $F$ is also elliptic).

Exactly the same arguments as in Theorems 1 and 2 yield

Corollary 3. If $d: X \rightarrow \Lambda^{\geq k} X$ in a Sullivan model for $S$, then

$$
\operatorname{cat}_{0} S \geq(k-2) \operatorname{dim} X^{\mathrm{ev}}+\operatorname{dim} X^{\text {odd }} \text {. }
$$


Examples 1-4 below show that the estimates of the corollaries can be sharp. We also present two examples (4 and 5), showing that $\iota_{n} \in Z(L)$ is not necessary, while the hypothesis $\left[\iota_{n}, \iota_{n}\right]=0$ is not sufficient. In our final example, the conclusion of Theorems 1 and 2 holds, but $\iota_{n}$ is not central and Poincaré duality is absent. However, the fibre is coformal.

The actual computation of rational L-S category in examples $1-5$ is carried out using the Mapping theorem [FH], the rational Ganea conjecture [J2], [He], the equality cat $_{0}=\mathrm{e}_{0}$ FHL, and simple arguments about the length of a representative of the top class. In all these cases, the minimal models are pure towers, i.e. 2-stage models with $d\left(X^{\mathrm{ev}}\right)=0$ and $d: X^{\text {odd }} \rightarrow \Lambda X^{\mathrm{ev}}$. In this situation, there is a second grading, namely the lower grading $(\Lambda X)_{p}:=\Lambda X^{\mathrm{ev}} \otimes \Lambda^{p} X^{\text {odd }}$, and we use the fact that Cartan $[\mathrm{C}]$ showed that the top class has lower degree $\operatorname{dim} X^{\text {odd }}-\operatorname{dim} X^{\mathrm{ev}}$. (A detailed proof is given in GHV, Chapter 2].) Moreover, we also rely on the fact that for an elliptic space $(\Lambda X, d)$, the formal dimension $N$, i.e. the largest $n$ for which $H^{n}(\Lambda X, d) \neq 0$, is given by [H1, p.188]

$$
N=\operatorname{dim} X^{\mathrm{ev}}-\sum_{i=1}^{\operatorname{dim} X}(-1)^{\left|x_{i}\right|}\left|x_{i}\right| .
$$

Example 1. Consider the model $\Lambda\left(a, b, y_{1}, y_{2}, y_{3} ; d\right)$ where $a$ and $b$ are of even degree, $d y_{1}=a^{2}, d y_{2}=b^{3}$ and $d y_{3}=a b^{2}$. Here, $\operatorname{dim} L^{\text {even }}=3, \operatorname{dim} Z L^{\text {odd }}=1$ (generated by " $b$ "), and cat $_{0}=4$, which is the estimate of Corollary 1 .

Example 2. Consider $\Lambda\left(a, b, y_{1}, y_{2}, y_{3} ; d\right)$ where $d y_{1}=a^{3}, d y_{2}=b^{3}$ and $d y_{3}=a^{2} b$. Here, the homotopy Lie algebra is abelian, and cat $_{0}=5=\operatorname{dim} L$, the lower bound guaranteed by Corollary 2 .

Example 3. To illustrate Corollary 3 , consider $\Lambda(a, y ; d)$ where $d y=a^{k}$ and $k \geq 2$. Here, cat $_{0}=k-1=(k-2) .1+1$.

Indeed, the bound of Corollary 3 is also sharp in many non-formal examples. In a pure tower where the even and odd dimensional homotopy are both concentrated in a fixed degree, the estimate is exact: If $X^{\mathrm{ev}}=X^{2 D}$ has dimension $p$ and $X^{\text {odd }}=$ $X^{2 k D-1}$ has dimension $q, d: X \rightarrow \Lambda^{k} X$, so Corollary 3 yields cat $0 p(k-2)+q$. On the other hand, the formal dimension is $N=p-q+2 D(q k-p)$, and the top class has lower degree exactly $q-p$. But $(\Lambda X)_{q-p}^{N}=\Lambda^{q-p} X^{\text {odd }} \otimes \Lambda^{p k-p} X^{\mathrm{ev}}$, as a short calculation shows. Hence, all representatives of the top class have the same length, so we can conclude that cat $_{0}=(q-p)+(p k-p)=p(k-2)+q$, as required.

Example 4. To see that the hypothesis $\iota \in Z(L)$ is not necessary for the conclusion of Theorem 1, and that the bounds of Theorem 2 can be sharp, consider the model $A=\Lambda\left(a, b, y_{1}, y_{2}, y_{3} ; d\right)$ where $d y_{1}=a^{3}, d y_{2}=b^{2}$ and $d y_{3}=a b$. Here, if $\iota_{x}$ denotes the element in $L_{A}$ corresponding to the generator $x$, we see that $\left[\iota_{a}, \iota_{b}\right] \neq 0$, and $\left[\iota_{a}, \iota_{a}\right]=0$. Short computations show that cat $_{0} A=4$ while cat $_{0}$ of the fibre by $\Lambda a$, namely $\Lambda\left(b, y_{1}, y_{2}, y_{3} ; \bar{d}\right)$, is 3 , agreeing with the estimate of Theorem 2 .

Example 5. On the other hand, $\left[\iota_{a}, \iota_{a}\right]=0$ is it not sufficient by itself, for consider $\Lambda(a ; 0) \rightarrow \Lambda\left(a, b, y_{1}, y_{2}, y_{3} ; d\right) \rightarrow \Lambda\left(b, y_{1}, y_{2}, y_{3} ; \bar{d}\right)$, where $d y_{1}=a^{3}, d y_{2}=b^{3}$ and $d y_{3}=a b$. Here, if $|a|=|b|=2$, the fibre is (rationally) $\mathbf{C} P^{2} \times \mathbf{S}^{5} \times \mathbf{S}^{3}$, which has category 4 . The formal dimension of the total space is 11 , so if its category were 5 , this would require the top class to have a representative of the form $\alpha y_{3}$, where $\alpha$ is a polynomial in $a$ and $b$. But this is not a cycle unless $\alpha=0$, so the category of the total space is at most 4 . However, the Mapping theorem guarantees at least 
the category of the fibre, which is 4 , so in this example the category of the total space is the same as that of the fibre.

Example 6. Finally, to show that neither Poincaré duality nor any assumption on the centre of the homotopy Lie algebra past $[\iota, \iota]=0$ is necessary, let $X$ be a space of category $n$ and let the homotopy fibre of the collapse of the suspension in $X \vee \Sigma Y \stackrel{c}{\rightarrow} X$ be $F$. Using the cube lemma [M] (the utility of which was patiently and expertly illustrated to the seond author by Octav Cornea), one computes the fibre and finds that $F \simeq \Sigma(\Omega X \times Y / \Omega X)$. In particular, when $Y=\mathbf{S}^{m-1}, F \simeq$ $\Omega X \times \mathbf{S}^{m} / \Omega X \simeq \Sigma^{m}\left(\Omega X \times \mathbf{S}^{0} / \Omega X\right)=\Sigma^{m}\left(\Omega X \vee \mathbf{S}^{0}\right)=\Sigma^{m} \Omega X \vee \mathbf{S}^{m}$

In any case, $F$ is a suspension and so cat $F=1$. Thus, if $X$ is not contractible, in the fibration $F \rightarrow X \vee \mathbf{S}^{2} \stackrel{c}{\rightarrow} X$ we have $\operatorname{cat}\left(X \vee \mathbf{S}^{2}\right)=\operatorname{cat} X$ while $\operatorname{cat} F=1$.

Now we specialize to the case when $X=\mathbf{C P}^{n}, n \geq 2$, where there is an auxiliary fibration $\mathbf{S}^{2 n+1} \rightarrow \mathbf{C} \mathbf{P}^{n} \rightarrow K(\mathbf{Z}, 2)$ defined by a generator of $H^{2} \mathbf{C} \mathbf{P}^{n}$. Consider the composition $\mathbf{C P}^{n} \vee \mathbf{S}^{2} \stackrel{c}{\rightarrow} \mathbf{C P}^{n} \rightarrow K(\mathbf{Z}, 2)$, and define $H$ by

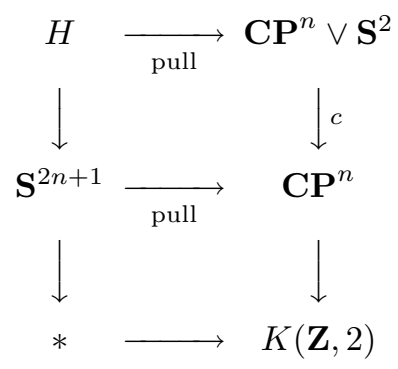

where "pull" denotes the pullback.

We compute $H$ as before, and find that

$$
H=\mathbf{S}^{2 n+1} \vee\left(\mathbf{S}^{1} \times \mathbf{S}^{2} / \mathbf{S}^{1}\right)=\mathbf{S}^{2 n+1} \vee \mathbf{S}^{3} \vee \mathbf{S}^{2},
$$

and so cat $H=1$. Hence, in the fibration (with zero connecting homomorphism)

$$
H \rightarrow \mathbf{C P}^{n} \vee \mathbf{S}^{2} \rightarrow K(\mathbf{Z}, 2)
$$

we have $n=\operatorname{cat}\left(\mathbf{C P}^{n} \vee \mathbf{S}^{2}\right) \geq \operatorname{cat} H+1=2$, while neither fibre nor total space is a Poincaré duality complex. Moreover, computations at the level of minimal models show that the Whitehead product of the fundamental class of $K(\mathbf{Z}, 2)$ and that of the $\mathbf{S}^{2}$ summand in the total space is the class represented by the $\mathbf{S}^{3}$ summand in the fibre, and hence is not zero. That is, the fundamental class is not in the centre of the homotopy Lie algebra of $\mathbf{C P}^{n} \vee \mathbf{S}^{2}$. Note, however, that since $H$ is a wedge of spheres, it is coformal.

Finally, we make some remarks about this example to illustrate that this increase in category from the fibre to the total space does not occur for 'other' reasons, in particular because of [J] applied to a locally nilpotent action in some associated fibration. Suppose $\mathbf{C P}^{n} \vee \mathbf{S}^{2} \rightarrow B$ is a fibre map (with zero connecting homomorphism) collapsing the sphere to a finite Postnikov piece $B$ of $\mathbf{C P}^{n}$ with fibre $F(B)$, necessarily non-contractible. We would then have a commutative diagram 
of pullbacks

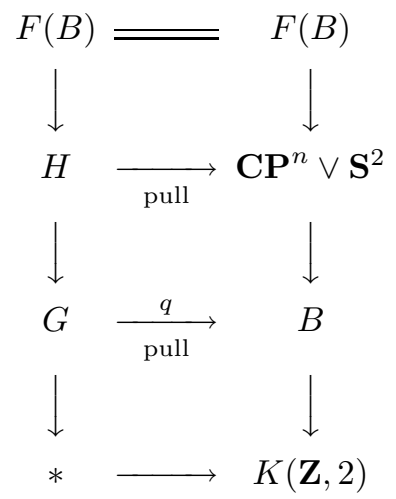

In particular, $F(B) \rightarrow H \rightarrow G$ is a fibration with zero connecting homomorphism.

Now, if $0 \neq \pi_{\text {odd }}(B) \otimes \mathbf{Q}$ (necessarily $=\pi_{2 n+1}(B) \otimes \mathbf{Q}$ ), then $0 \neq \pi_{\text {odd }}(G) \otimes \mathbf{Q}$ as well, since $q$ induces an isomorphism in odd dimensional homotopy groups. If an element $u \in \pi_{\text {odd }}(G) \otimes \mathbf{Q}$ acted locally nilpotently on the homology of the fibre $F(B)$, we'd have $\operatorname{cat}_{0}(H) \geq 1+\operatorname{cat}_{0} F(B) \geq 2$ by [J]. Since cat $H=1$, this cannot occur, and so $\operatorname{cat}_{0} F(B)=\operatorname{cat}_{0}(H)=1$ also.

The naturality of the holonomy action implies that the action of $q_{*}(u)$ on $H_{*} F(B)$ cannot be locally nilpotent either, so the fact that the L-S category of $\mathbf{C P}^{n} \vee \mathbf{S}^{2}$ is strictly greater than that of $H$ cannot be deduced from any local nilpotence in the holonomy action in the fibration

$$
F(B) \rightarrow \mathbf{C P}^{n} \vee \mathbf{S}^{2} \rightarrow B
$$

and the fact that $\operatorname{cat}_{0} F(B)=\operatorname{cat}_{0}(H)$ : it is 'essentially' due to the even dimensional homotopy in $B$.

\section{REFERENCES}

[BG] A.K. Bousfield and V.K.A.M. Gugenheim, On PL De Rham theory and rational homotopy type, Mem. Amer. Math. Soc. 8 (1976). MR 54:13906

[C] H. Cartan, La transgression dans un groupe de Lie et dans un espace fibré principal, Colloque de Topologie (espaces fibrés), Bruxelles (1950), Thone, Liège (1951), 55-71. MR 13:107f

[F] Y. Félix, La Dichotomie Elliptique-Hyperbolique en Homotopie Rationnelle, Astérisque 176 (1989). MR 91c:55016

[FH] Y. Félix and S. Halperin, Rational L.-S. category and its applications, Trans. Amer. Math. Soc. 273 (1982), 1-38. MR 84h:55011

[FHL] Y. Félix, S. Halperin and Jean-Michel Lemaire, The rational LS category of products and Poincaŕe duality complexes, Topology 37, No. 4 (1998), 749-756. MR 99a:55003

[FHT] Y. Félix, S. Halperin, and J.-C. Thomas,, The homotopy Lie algebra for finite complexes, Inst. Hautes Études Sci. Publ. Math 56 (1982), 179-202 (1983). MR 85c:55010

[FHT1] _ Gorenstein Spaces, Advances in Mathematics 71 (1988), no. 1, 92-112. MR 89k:55019

[GHV] W. Greub, S. Halperin and R. Vanstone, Connections, Curvature and Cohomology, VIII, Academic Press, Inc., 1976. MR 53:4110

[G] P.P. Grivel, La suite spectral d'une fibration de Kan, C.R.Acad. Sci. Paris 282 (1976), 1227-1229. MR 53:14494

[H] S. Halperin, Lectures on Minimal Models, Mém. Soc. Math France 9-10 (1983). MR 85i:55009

[H1] Finiteness in the Minimal Models of Sullivan, Trans. Amer. Math. Soc. 230 (1977), 173-199. MR 57:1493 
[He] Kathryn P. Hess, A Proof of Ganea's Conjecture for Rational Spaces, Topology 30 (2) (1991), 205-214. MR 92d:55012

[J1] Barry Jessup, L.-S. category and homogeneous spaces, Journal of Pure and Applied Algebra 65 (1990), 45-56. MR 91e:55004

[J2] , Rational L.-S. category and a conjecture of Ganea, Journal of Pure and Applied Algebra 65 (1990), 57-67. MR 91e:55005

[J] Holonomy-Nilpotent Fibrations and Rational Lusternik-Schnirelmann Category, Topology 34 (4) (1995), 759-770. MR 96k:55014

[M] M. Mather, Pull-backs in homotopy theory, Canad. J. Math. 28 (1976), 225-263. MR 53:6510

[S] D. Sullivan, Infinitesimal computations in topology, Inst. Hautes Études Sci. Publ. Math 47 (1978), 269-331. MR 58:31119

[T] G.H. Toomer, Lusternik-Schnirelmann category and the Moore spectral sequence, Math. Z. 138 (1974), 123-143. MR 50:8509

[W] George W. Whitehead, Elements of Homotopy Theory, Graduate Texts in Mathematics, vol. 61, Springer-Verlag, Berlin-Hedelberg-New York, 1978. MR 80b:55001

Institut Préparatoire aux Études d'ingénieurs de Sousse, Tunisie

E-mail address: Sonia.Ghorbal@ipeiss.rnu.tn

Department of Mathematics and Statistics, University of Ottawa, Ottawa, Ontario, Canada K1N 6N5

E-mail address: bjessup@uottawa.ca 